\title{
Predicción ecotoxicológica ante concentraciones no deseadas de cianuro libre en efluentes mineros-auríferos
}

Eco toxicological prediction of undesirable concentrations of free cyanide in mineral-aurifer effluents

Recibido: agosto 22 de 2017 | Revisado: setiembre 21 de 2017 | Aceptado: octubre 26 de 2017

\author{
René G. Sosa VILCA ${ }^{1}$ \\ George Argota Pérez ${ }^{2}$
}

\section{RESUMEN}

El objetivo del estudio fue predecir la ecotoxicología ante concentraciones no deseadas de cianuro libre en efluentes mineros-auríferos. Se seleccionó durante el período de lluvia, cuatro muestras de efluentes donde se determinóla concentración de cianuro libre mediante el método del nitrato de plata. Con la mayor concentración se realizó el bioensayo de toxicidad letal aguda utilizando el modelo biológico de experimentación Eisenia andrei y con ambas determinaciones se estimó, la predicción ecotoxicológica, a través del modelo computacional Gecotoxic. Las concentraciones de las muestras de los efluentes fueron: $0,161 \pm 0,008 ; \quad 0,142 \pm 0,008 ; \quad 0,138 \pm 0,008$ y $0,137 \pm 0,007$ mg. $\mathrm{L}^{-1}$, superándose el valor permisible por la norma ambiental utilizada $\left(0,022 \mathrm{mg} \cdot \mathrm{L}^{-1}\right)$. La $\mathrm{CL}_{50}$ calculada para efluentes fue de 0,078 mg. $\mathrm{L}^{-1}$, observándose efectos letales mientras que, el modelo computacional Gecotoxic indicó, predicción de riesgo ambiental de tipo alta (68\%). Se concluyó que, los efluentes mineros se vertieron con valores no deseados de cianuro libre que pueden ser perjudiciales para los cuerpos receptores.

Palabras clave: actividad aurífera, toxicidad, cianuro libre, efluentes, riesgo ecotoxicológico

\section{Abstract}

The objective of this work was to predict the ecotoxicology of unwanted concentrations of free cyanide in mining-gold effluents. Four samples of effluents were selected during the rainy season, where the concentration of free cyanide was determined by the silver nitrate method. With the highest determined concentration, a bioassay of acute lethal toxicity was performed using the Eisenia andrei biological model of experimentation and with both determinations the ecotoxicological prediction was estimated through the Gecotoxic computer model. The concentrations of the samples of the effluents were: $0.161 \pm$ $0.008 ; 0.142 \pm 0.008 ; 0,138 \pm 0,008$ and $0,137 \pm 0,007$ mg.L1 , exceeding the allowable value by the environmental standard used (0,022 mg.L-1). The LC50 calculated for effluents was $0.078 \mathrm{mg} . \mathrm{L}-1$, with lethal effects observed, while the Gecotoxic computational model indicated high-level environmental risk prediction (68\%). It was concluded that the mining effluents were discharged with unwanted concentrations of free cyanide, which could be harmful to the receiving water bodies.

Key words: gold activity, toxicity, free cyanide, effluents, ecotoxicological risk
1 Universidad Nacional de Moquegua, Perú

gersovil@hotmail.com.com

2 Centro de Investigaciones Avanzadas y formación Superior en Educación, Salud y Medio Ambiente "AMTAWI", Perú george.argota@gmail.com 


\section{Introducción}

La actividad minera, a través de los siglos, ha formado parte de la historia y del desarrollo económico de muchos países a nivel mundial. La extracción de metales nobles como el oro, empezó a incrementarse en el siglo XVI después del descubrimiento de las Américas (Gallardo et al.; 2013). Sin embargo, la extracción y procesamiento del mineral aurífero pueden degradar los entornos naturales como el suelo, agua, calidad del aire, además de la salud humana (Saldarriaga et al., 2013; Ako et al., 2014). La contaminación se produce en diferentes etapas e incluye el carbón en lixiviación, lixiviación en pilas con cianuro, oxidación biológica y el tostado (Leder et al., 2012). En particular, existe una baja recuperación de oro con excesivas concentraciones o consumo de cianuro, no siendo viable para la extracción del mineral aurífero (Oraby, Eksteen \& Tanda, 2017).

Varios estudios se refieren al impacto dañino por exposición al cianuro sobre la salud humana debido a la extracción del oro (Graff \& Neidell, 2013; Currie et al., 2014), siendo limitada la predicción de riesgo con base a biomodelos de experimentación y modelos computacionales. Deloya (2012) explica que verter residuos líquidos con concentraciones de cianuro sin ningún tratamiento, sobrepasará la capacidad de auto-recuperación de los cuerpos receptores; es decir, la minería se encuentra entre las que producen mayores impactos antropogénicos (Cordy et al., 2011; Módenes et al., 2012).

Actualmente, toda metodología de evaluación de riesgo debe ser requerida bajo un enfoque de gestión que permita la predicción sobre determinado com- portamiento (Pérez \& Valero, 2013) y en este sentido, los múltiples resultados bajo pruebas de campo con medición real de indicadores (incluyendo minería de datos), resulta necesario (Gunvig, Hansen \& Borggaard, 2013).

El presente estudio tiene como objetivo predecir la ecotoxicología ante concentraciones no deseadas de cianuro libre en efluentes mineros-auríferos.

\section{Materiales y métodos}

El estudio se realizó durante el periodo de lluvia donde se recolectó cuatro muestras en efluentes y relaves mineros procedentes de la actividad aurífera para la determinación de cianuro libre y cianuro WAD. El método de determinación de cianuro libre $\left(\mathrm{CN}^{-}\right)$fue por valoración con $\mathrm{AgNO}_{3}$, estado basado en la formación del ion complejo $\mathrm{Ag}(\mathrm{CN})_{2}{ }^{-}$el cual es muy estable e incoloro.

$$
\begin{aligned}
& \cdot 2 \mathrm{CN}^{-}+\mathrm{Ag}^{+} \mathrm{NO}_{3}^{-} \longrightarrow \mathrm{Ag}(\mathrm{CN})_{2}^{-} \\
& \cdot \mathrm{AgNO}_{3}+2 \mathrm{NaCN} \rightarrow \mathrm{NaAg}(\mathrm{CN})_{2}+\mathrm{NaNO}_{3}
\end{aligned}
$$

$\mathrm{Al}$ acomplejarse todo el ion cianuro presente y existir un pequeño exceso de $\mathrm{Ag}+$ añadido, fue detectado por el indicador 5-(4-dimetilaminobencilideno)-Rodanina, que es sensible a la plata cambiando inmediatamente de amarillo a color anaranjado. A medida que se añadió $\mathrm{AgNO}_{3}$ (gota a gota desde la bureta) se consumió el cianuro en disolución para formarse $\mathrm{NaAg}(\mathrm{CN})_{2}$. Luego, comenzó a reaccionar el complejo formado con las gotas de rodanina añadidas, creando un compuesto rosado. Al existir sulfuros en la muestra se añadió, $5 \mathrm{ml}$ de la solución $0,5 \mathrm{M}$ de nitrato de cadmio. 
Se dejó sedimentar el precipitado amarillo de sulfuro de cadmio y mediante un papel de filtro de porosidad media, se filtró el sobrenadante. Se lavó el papel y el residuo con agua caliente dos veces. Posteriormente, se añadió al filtrado $0,5 \mathrm{ml}$ de la solución del indicador Rodanina donde se observó un cambio de coloración. Para confirmar el cambio, se valoró paralelamente una solución en blanco que contuvo las mismas cantidades de agua e indicador que las utilizadas en la muestra cuyos resultados se presentaron mediante la expresión: $\mathrm{NaCN}(\mathrm{ppm})=$ $(\mathrm{a}-\mathrm{b}) / \mathrm{V}$ alícuota $* 1000$

La determinación de la curva dosis-respuesta $\left(\mathrm{CL}_{50}\right)$ se realizó mediante el modelo biológico de experimentación $E i$ senia andrei donde se siguió lo recomendado por la guía 207 (OCDE, 1984) y para lo cual, se establecieron dos réplicas con cuatro tratamientos (Figura 1).

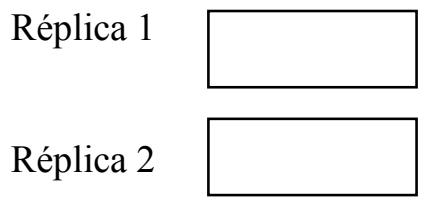

Control

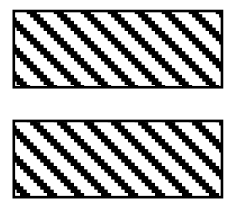

$\mathrm{X} / 1$

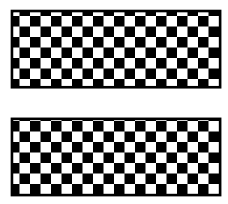

$\mathrm{X} / 2$

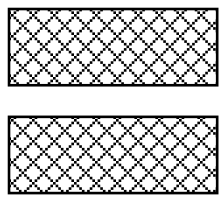

$\mathrm{X} / 3$

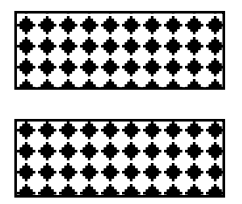

$\mathrm{X} / 4$

Figura 1. Tratamientos en el modelo biológico de experimentación para la curva dosis-respuesta

Los datos de concentración/mortalidad se utilizaron para calcular la concentración letal media $\left(\mathrm{CL}_{50}\right)$, la cual se determinó por el método Probit, según la siguiente expresión: $\mathrm{p}=(\mathrm{r} / \mathrm{n}) \times 100$. Donde: número de individuos $(n)$, número de organismos muertos o afectados $(r)$ y porcentaje de efecto $(p)$.

El método probit es una transformación sobre la tasa de efecto $(p)$ y la ecuación generada es de forma lineal $\mathrm{y}=\mathrm{a}+\mathrm{bx}$. Donde y (expresado en unidades probit) $=\mathrm{z}+5, \mathrm{z}=$ variable normal estándar $=\mathrm{z}_{0}$ tal que la Prob $\left(\mathrm{z} \leq \mathrm{z}_{0}\right)=\mathrm{p}$, a y b son los estimadores de los parámetros de la recta de regresión, Cuando $\mathrm{p}=50 \%$ entonces $y=5$, por tanto: $x_{5}=\log 10 \mathrm{CL}_{50}$.

Finalmente, la predicción ecotoxicológica para estimar el riesgo fue mediante el modelo computacional Gecotoxic, el cual es un analizador multifuncional para residuales ambientales que es operativo en datos reales a partir de la interacción matricial de números codificados arábigamente por intervalos de puntuación (Argota \& Iannacone, 2014).

Los datos fueron estudiados mediante el análisis de la varianza con réplicas para definir las fuentes de variación significativas. Se utilizó el software Statgraphics Centurion XVI, en el que se consideró la prueba de contraste múltiple de rango, mediante el test de Bonferroni para comparar las mediciones analíticas. A través del test Kolmogorov-Smirnov de bondad de ajuste, se comprobó la normalidad de los datos, considerándolos significativos con $\mathrm{p}<0,05$.

\section{Resultados y discusión}

La Tabla 1 muestra las concentraciones de cianuro libre en efluentes y relaves, las cuales superaron los valores recomendados $\left(0,022 \mathrm{mg} . \mathrm{L}^{-1}\right)$ lo cual indicó 
que, los efluentes fueron descargados en concentraciones significativas. Además, hubo diferencias estadísticas entre las concentraciones de las muestras analizadas (Tabla 2).

Tabla 1

Concentración de cianuro libre en efluentes (mg. $\left.L^{-1}\right)$

\begin{tabular}{l|cccc}
\hline \multirow{1}{*}{ Residuos } & \multicolumn{4}{c}{ Muestra } \\
\cline { 2 - 5 } & 1 & 2 & 3 & 4 \\
\hline efluentes & $0,161 \pm 0,008$ & $0,142 \pm 0,008$ & $0,138 \pm 0,008$ & $0,137 \pm 0,007$ \\
referencia* & & \multicolumn{3}{|c}{0,022} \\
\hline
\end{tabular}

*: Estándares de Calidad de Agua (uso IV). Ley General de Recursos Hídricos: Ley No. 29338.

Tabla 2

Análisis de la varianza y homogeneidad ante las concentraciones de cianuro libre

\begin{tabular}{cccccc}
\hline FV & SC & gl & CM & Coeficiente-F & Valor-P \\
\hline Entre grupos & 0,001131 & 3 & 0,000377 & 377,00 & 0,0000 \\
Intra grupos & 0,000008 & 8 & 0,000001 & & \\
Total (Corr.) & 0,001139 & 11 & & & \\
\hline
\end{tabular}

Prueba de múltiple rango

\begin{tabular}{ccc}
\hline muestra & media & $\begin{array}{c}\text { grupos } \\
\text { homogéneos }\end{array}$ \\
\hline 4 & $0,137 \pm 0,007$ & a \\
3 & $0,138 \pm 0,008$ & a \\
2 & $0,142 \pm 0,008$ & b \\
1 & $0,161 \pm 0,008$ & c \\
\hline
\end{tabular}

La Figura 2 muestra la curva dosis-respuesta en el modelo biológico de experimentación Eisenia andrei por exposición a cianuro libre. $\mathrm{Al}$ exponer los ejemplares a experimentación durante las primeras 24 horas se observó, mortalidad del 100 $\%$ de los individuos $\left(\mathrm{CL}_{50}\right)$ y es significa- tivo porque este tipo de test establece hasta un máximo de duración de 96 horas. La exposición de los ejemplares al 50\% del efluente diluido $\left(0,078 \mathrm{mg} \cdot \mathrm{L}^{-1}\right)$ se produjo el $50 \%$ de muerte, lo cual indicó una alta toxicidad de los efluentes. 

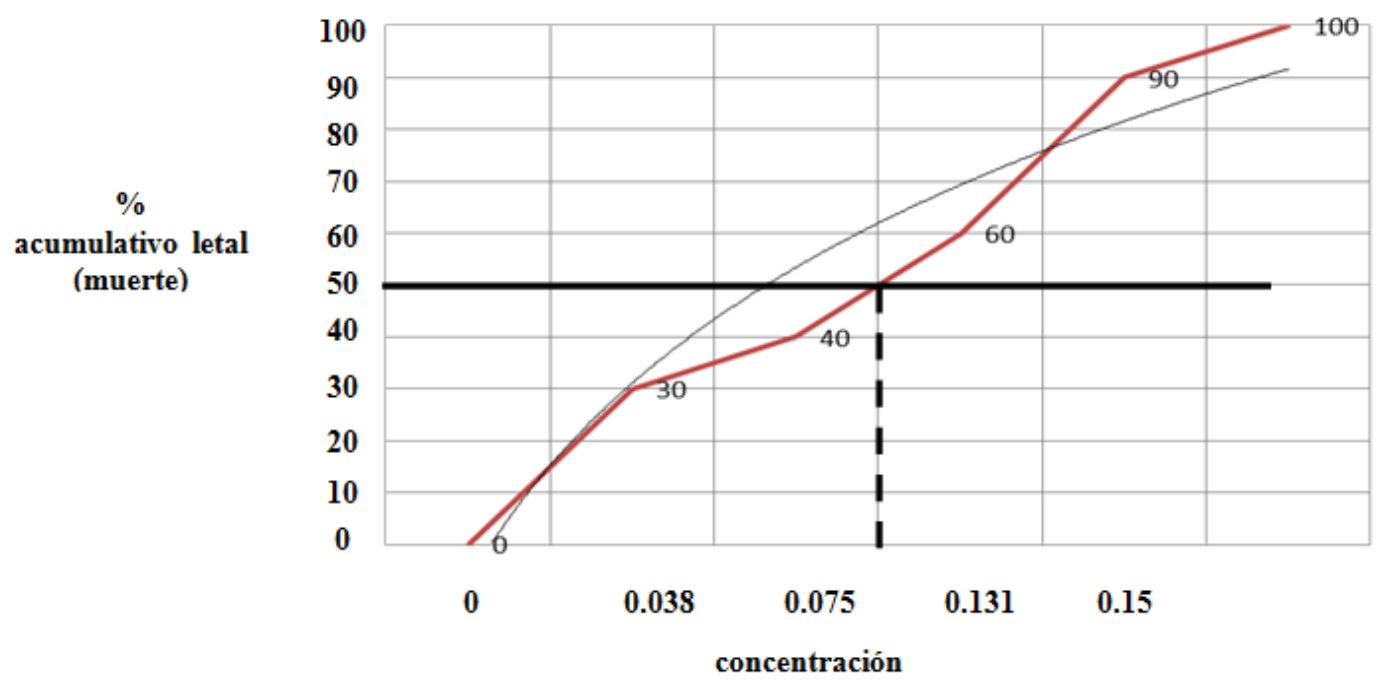

Figura 2. Curva dosis-respuesta en Eisenia andrei por exposición a cianuro libre

La Figura 3 muestra la predicción de riesgo ecotoxicológica estimada por el modelo computacional Gecotoxic.
El cianuro puede ser sometido a una degradación química, natural o biológica para disminuir su poder contaminante (Wotruba et al., 1988).

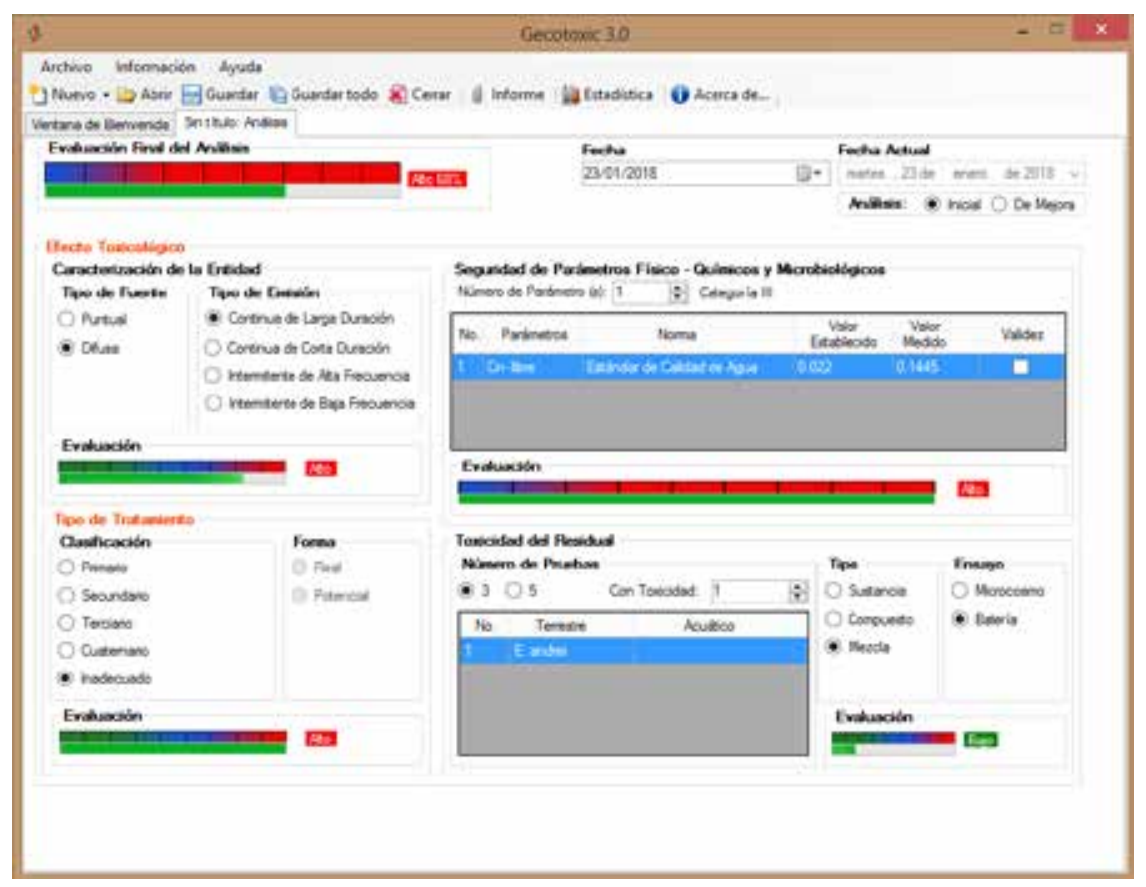

Figura 3. Predicción ecotoxicológica mediante el modelo computacional Gecotoxic

Dentro de las clasificaciones del cianuro, está el cianuro total, cianuro disociable en ácido débil (WAD) y el cianuro libre, que fue la variable obje- to en este estudio y para que no existan riesgos ambientales, las concentraciones deben estar en orden de seguridad y regulación estequiométrica (Figura 2). 
Los resultados (Figura 2) mostraron que hubo concentraciones no deseadas de cianuro libre en los efluentes (Domínguez, Velando \& Ferreiro, 2005). De esta manera, se observó que en la relación dosis-respuesta es importante destacar que el uso de bioensayos posibilita evaluar la calidad de residuos y del ambiente (Kudłak et al., 2012; Farré et al., 2013).

El modelo computacional Gecotoxic indicó, según la interacción entre la caracterización del residual, la determinación de cianuro libre, toxicidad con el biomodelo y tipo de tratamiento sobre la fuente, que la predicción estimada del riesgo fue alta (68\%) lo que señala la problemática ambiental sobre cualquier actividad minera aurífera cuando sus efluentes contienen cianuros no tratados.

\section{Conclusiones}

Los efluentes mineros se vertieron con valores no deseados de cianuro libre siendo perjudicial para los cuerpos acuáticos receptores, además, a la salud animal y humana ante cualquier procesos de transferencia dado el uso de aguas contaminadas.

\section{Referencias}

Ako, T.A., Onoduku, U.S., Oke, S.A., Adamu, I.A., Ali, S.E. \& et al. (2014). Environmental impact of artisanal gold mining in Luku, Minna, Niger State, North Central Nigeria. Journal of Geosciences and Geomatics; 2(1), 28-37. Doi: 10.12691/jgg-2-1-5. Recuperado en: file://C:/Users/CLIENTE/ Downloads/jgg-2-1-5.pdf

Argota, P.G. \& Iannacone, O.J. (2014). Metodología informatizada GECOTOX para la predicción de riesgo ecotoxicológico por exposición a efectos contaminantes en efluentes ambientales y ecosistemas acuáticos. The Biologist; 12(2), 181-193.

Cordy, P., Veiga, M.M., Salih, I., Al-Saadi, S., Console, S., Garcia, O. \& et al. (2011). Mercury contamination from artisanal gold mining in Antioquia, Colombia: the world's highest per capita mercury pollution. Sci Total Environ; 410-411,
154-160. Doi: 10.1016/j.scitotenv.2011.09.006.

Currie, J., Zivin, J.S.G., Mullins, J. \& Neidell, M.J. (2014). What do we know about short- and long-term effects of early-life exposure to pollution?. Ann Rev Resour Econ; 6, 217-247. Recuperado en: https:// doi.org/10.1146/annurev-resource-100913-012610

Deloya, M.A. (2012). Tratamiento de desechos del cianuro por biorremediación. Tecnología en marcha. 25(2), 61-72. Recuperado en: file://C:/Users/CLIENTE/Downloads/Dialnet-TratamientoDeDesechosDelCianuroPorBiorremediacion-4835624.pdf

Domínguez, J., Velando, A. \& Ferreiro, A. (2005). Are Eisenia fétida (Savigny, 1826) and Eisenia andrei Bouche (1972) (Oligochaeta, Lumbricidae) differentt biological spe- 
cies?. Pedobiologia; 49, 81-87. Doi: 10.1016/j.pedobi.2004.08.005. Recuperado en: http://www.scielo. org. mx/pdf/azm/v26nspe2/v26nspe2a24.pdf

Farré, M.J., Day, S., Neale, P.A., Stalter, D., Tang, J.Y.M. \& Escher, B.I. (2013). Bioanalytical and chemical assessment of the disinfection by-product formation potential: role of organic matter. Water Res; 47 , 5409-5421. Doi: 10.1016/j.watres.2014.04.002. Recuperado en. https://espace.library.uq.edu.au/ view/UQ:331090/UQ331090OA. pdf

Gallardo, M.D., Cabrera, D.I., Bruguera, A.N. \& Madrazo, E.F. (2013). Evaluación de impactos ambientales provocados por la actividad minera en la localidad de Santa Lucía, Pinar del Río. Revista Avances; 15(1), 94-108. Recuperado en: http://www.ciget.pinar.cu/Revista/ No.2013-1/articulos/impactos_ ambientales_mineria.pdf

Graff, Z.J.S. \& Neidell, M.J. (2013). Environment, health, and human capital. J Econ Lit; 51(3), 689-730. Doi: 10.1257/jel.51.3.689.

Gunvig, A., Hansen, F. \& Borggaard, C. (2013). A mathematical model for predicting growth/ no-growth of psychrotrophic C. botulinum in meat products with five variables. Food Control; 29, 309-317. Recuperado en: http://dx.doi.org/10.1016/j.foodcont.2012.06.046
Kudłak, B., Rogowska, J., Wolska, L., Kałas, M. \& Łęczyński, L.J.N. (2012). Toxicity assessment of sediments associated with the wreck of s/s Stuttgart in the Gulf of Gdańsk (Poland). J Environ Monit; 14, 1231-1236.

Leder, O.M., Hessen, D.O., Lindberg, Y., Nystuen, G. \& Rathe, B. (2012). Recommendation to Exclude AngloGold Ashanti Limited From the Investment Universe of the Government Pension Fund Global, in Annual Report 2013, Council on Ethics for the Government Pension Fund Global, 94-135. Recuperado en: http://etikkradet.no/files/2014/12/Annual_ report_2013.pdf

Módenes, A.N., Espinoza, Q.F.R., Borba, F.H. \& Manenti, R.D. (2012). Performance evaluation of an integrated photo-Fenton - electrocoagulation, process applied to pollutant removal from tannery effluent in batch system. Chem Eng J; 197, 1-9. Doi: 10.1016/j. cej.2012.05.015

Oraby, E.A., Eksteen, J.J. \& Tanda, B.C. (2017). Gold and copper leaching from gold-copper ores and concentrates using a synergistic lixiviant mixture of glycine and cyanide. $H y$ drometallurgy; 169, 339-345. Doi: 10.1016/j.hydromet.2017.02.019

Pérez, R.F. \& Valero, A. (2013). Software and Data Bases: Use and Application. Predictive Microbiology in Foods. In: Springer Briefs in Food, Health and Nutrition, 5, 75-85. 
Saldarriaga, I.A., Villegas, P.C. \& Arango, S. (2013). The public good dilemma of a non-renewable common resource: a look at the facts of artisanal gold mining. Res Policy; 38 (2), 224-232.
Wotruba, H., Hruschka, F., Hentschel, T. \& Priestel, M. (1998). Manejo ambiental en la pequeña minería. MEDMIN-COSUDE, La Paz-Bolivia, Pp. 155. Recuperado en: http:// www.gama-peru.org/libromedmin/ 